\title{
PARP-1-Targeted Radiotherapy in Mouse Models of Glioblastoma
}

\author{
Stephen A. Jannetti ${ }^{1-3}$, Giuseppe Carlucci ${ }^{3,4}$, Brandon Carney ${ }^{3,5,6}$, Susanne Kossatz ${ }^{3}$, Larissa Shenker ${ }^{3,7}$, \\ Lukas M. Carter ${ }^{3}$, Beatriz Salinas ${ }^{3}$, Christian Brand ${ }^{3}$, Ahmad Sadique ${ }^{3}$, Patrick L. Donabedian ${ }^{3}$, Kristen M. Cunanan ${ }^{8}$, \\ Mithat Gönen ${ }^{8}$, Vladimir Ponomarev ${ }^{3,7}$, Brian M. Zeglis ${ }^{1,3,7,9,10}$, Mark M. Souweidane ${ }^{11,12}$, Jason S. Lewis ${ }^{3,7,9,10}$, \\ Wolfgang A. Weber ${ }^{3,7,10}$, John L. Humm ${ }^{13}$, and Thomas Reiner ${ }^{3,10}$
}

${ }^{I}$ Department of Biochemistry, Hunter College-The City University of New York, New York, New York; ${ }^{2}$ Department of Biochemistry, The Graduate Center, The City University of New York, New York, New York; ${ }^{3}$ Department of Radiology, Memorial Sloan Kettering Cancer Center, New York, New York; ${ }^{4}$ Department of Radiology, Center for Advanced Imaging Innovation and Research, New York University Langone Medical Center, New York, New York; ${ }^{5}$ Department of Chemistry, The Graduate Center, The City University of New York, New York, New York; ${ }^{6}$ Department of Chemistry, Hunter College-The City University of New York, New York, New York; ${ }^{7}$ Molecular Pharmacology Program, Memorial Sloan Kettering Cancer Center, New York, New York; ${ }^{8}$ Department of Epidemiology and Biostatistics, Memorial Sloan Kettering Cancer Center, New York, New York; ${ }^{9}$ Department of Pharmacology, Weill-Cornell Medical College, New York, New York; ${ }^{10}$ Department of Radiology, Weill-Cornell Medical College, New York, New York; ${ }^{11}$ Department of Neurological Surgery, Weill-Cornell Medical College, New York, New York; ${ }^{12}$ Department of Neurosurgery, Memorial Sloan Kettering Cancer Center, New York, New York; and ${ }^{13}$ Department of Medical Physics, Memorial Sloan Kettering Cancer Center, New York, New York

The DNA repair enzyme poly(ADP-ribose) polymerase 1 (PARP-1) is overexpressed in glioblastoma, with overall low expression in healthy brain tissue. Paired with the availability of specific small molecule inhibitors, PARP-1 is a near-ideal target to develop novel radiotherapeutics to induce DNA damage and apoptosis in cancer cells, while sparing healthy brain tissue. Methods: We synthesized an ${ }^{131}$ I-labeled PARP-1 therapeutic and investigated its pharmacology in vitro and in vivo. A subcutaneous tumor model was used to quantify retention times and therapeutic efficacy. A potential clinical scenario, intratumoral convection-enhanced delivery, was mimicked using an orthotopic glioblastoma model combined with an implanted osmotic pump system to study local administration of ${ }^{131}$ I-PARPi (PARPi is PARP inhibitor). Results: ${ }^{131} \mid-$ PARPi is a $1(2 \mathrm{H})$-phthalazinone, similar in structure to the Food and Drug Administration-approved PARP inhibitor AZD-2281. In vitro studies have shown that ${ }^{131}$ I-PARPi and AZD-2281 share similar pharmacologic profiles. ${ }^{131}$ I-PARPi delivered 134.1 cGy/ $\mathrm{MBq}$ intratumoral injected activity. Doses to nontarget tissues, including liver and kidney, were significantly lower. Radiation damage and cell death in treated tumors were shown by p53 activation in U87-MG cells transfected with a p53-bioluminescent reporter. Treated mice showed significantly longer survival than mice receiving vehicle (29 vs. $22 \mathrm{~d}, P<0.005$ ) in a subcutaneous model. Convection-enhanced delivery demonstrated efficient retention of ${ }^{131}$ I-PARPi in orthotopic brain tumors, while quickly clearing from healthy brain tissue. Conclusion: Our results demonstrate ${ }^{131} \mathrm{I}-$ PARPi's high potential as a therapeutic and highlight PARP's relevance as a target for radionuclide therapy. Radiation plays an integral role in brain tumor therapy, and radiolabeled PARP therapeutics could ultimately lead to improvements in the standard of care.

Received Nov. 9, 2017; revision accepted Mar. 5, 2018

For correspondence or reprints contact: Thomas Reiner, Department of Radiology, Memorial Sloan Kettering Cancer Center, 1275 York Ave., New York, NY 10065

E-mail: reinert@mskcc.org

Published online Mar. 23, 2018.

COPYRIGHT (C 2018 by the Society of Nuclear Medicine and Molecular Imaging.
Key Words: PARP; radiotherapeutic; ${ }^{131} \mathrm{I}-\mathrm{PARP}$; ${ }^{131} \mathrm{I}$; convection enhanced delivery (CED)

J Nucl Med 2018; 59:1225-1233

DOI: 10.2967/jnumed.117.205054

$\mathbf{G}$ lioblastoma is the most common primary brain tumor in adults, with more than 12,000 diagnoses per year (1). Current standard treatment consists of maximal surgical resection followed by chemotherapy (temozolomide) and external beam radiation. This, however, only minimally extends median survival (2), and most patients develop recurrent tumors within months $(3,4)$. The diffuse growth pattern is the fundamental reason why surgical resection and external beam radiotherapy are insufficient, because highly dispersed glioblastoma cells would require larger tumor margins to be treated and resected, which can result in unacceptably high healthy tissue loss and significant morbidities. A selective cellular-based approach would provide significant advantages over conventional therapy.

Recently, progress has been made toward the delivery of targeted therapeutics to central nervous system tumors, especially using convection enhanced delivery (CED) $(5,6)$. This strategy uses catheters to infuse therapeutics directly into the affected brain tissue, where they are distributed by convective rather than diffusive properties. This approach offers considerable advantages for delivery and tissue penetrance of therapeutic antibodies, virus vectors, and cell-based therapeutics. Using this technology, even radiolabeled antibodies, which normally have limited tissue penetration, can be delivered (7), potentially improving therapeutic efficacy. Nevertheless, because of the heterogeneous nature of the disease (8), further enhancement of delivery and tissue penetration is necessary, which could be achieved through CED of targeted small-molecule radiotherapeutics.

One target for use in CED and targeted radiation therapy is poly (ADP-ribose) polymerase (PARP-1). PARP-1 is a fundamentally 
important member of the cellular DNA repair machinery that highly proliferative cancer cells rely on to maintain genomic integrity through an accelerated cell cycle (9). PARP-1 expression was reported in the nucleoli of neurons, oligodentritic cells, and astrocytes as well as the Purkinje cell layer in the cerebellum and the dentate gyrus (10). Nevertheless, it has been shown that malignant glial growths have elevated PARP-1 expression compared with healthy pediatric and adult brain tissue (11), forming an ideal foundation for CED-based therapeutics. Similarly important, PARP inhibitors not only quickly distribute and bind within PARP-expressing cells, but also simultaneously washout effectively from healthy tissues, resulting in high target-to-background contrast $(10,12)$, potentially providing a large treatment window for CED therapy (7). Recently, advances have been made in PARP-targeted molecular imaging, and much of this work has focused on glioblastoma $(13,14)$ and other central nervous system tumors (10). Intuitively, replacing a fluorescent or PET active imaging tag with a radiotherapeutic isotope would take advantage of the high specificity exhibited by the PARP-targeted imaging agents as well as the high PARP expression seen in glioblastoma. More information on the relevant chemistry and PARP inhibitors used for imaging are provided in comprehensive reviews $(12,15)$.

In this study, we use ${ }^{131} \mathrm{I}-\mathrm{PARP}$, a small-molecule derived from an inhibitor screening library (14), and explore and develop its value as a CED agent for glioblastoma therapy. We found that the binding profile of ${ }^{131} \mathrm{I}$-PARPi matches that of other PARP inhibitors and imaging agents. In a mouse model of glioblastoma, we investigate the pharmacokinetics of ${ }^{131}$ I-PARPi and estimate the delivered radiotherapeutic doses. We show that p53 expression was activated by ${ }^{131} \mathrm{I}$-PARPi and that both single and fractionated doses extend the overall survival of tumor-bearing mice. In an orthotopic model of glioblastoma, we corroborate that high levels of ${ }^{131}$ I-PARPi accumulate via CED, whereas healthy brain tissue only retains the small molecule at low levels. Taken together, our results show that ${ }^{131} \mathrm{I}$-PARPi is a promising radiotherapeutic small molecule and could potentially improve glioblastoma therapy.

\section{MATERIALS AND METHODS}

\section{General}

Unless specified otherwise, all reagents were purchased from Sigma-Aldrich and used as received. ${ }^{131} \mathrm{I}-\mathrm{Na}$ in $0.1 \mathrm{~N} \mathrm{NaOH}$ with a specific activity more than 4,600 TBq/g was purchased from Nordion. 4-(4-fluoro-3-(4-(3-iodobenzoyl)piperazine-1-carbonyl)benzyl)phthalazin-1(2H)-one was synthesized as described previously (14). Olaparib (AZD-2281) was purchased from LC Laboratories. PARPi-FL was synthesized as previously described $(16,17)$. Water $(>18.2$ $\mathrm{M} \Omega \mathrm{cm}^{-1}$ at $25^{\circ} \mathrm{C}$ ) was obtained from an Alpha-Q Ultrapure water system (Millipore) and acetonitrile (AcN) as well as ethanol (EtOH) were of high-performance liquid chromatography (HPLC) grade purity. Sterile $0.9 \%$ saline solution (Hospira) was used for all in vivo injections. HPLC purification and analysis were performed on a Shimadzu UFLC HPLC system equipped with a DGU-20A degasser, a SPD-M20A UV detector, a LC-20AB pump system, and a CBM-20A Communication BUS module. A LabLogic Scan-RAM radio-thinlayer chromatography/HPLC detector was used to detect activity. HPLC solvents (buffer A: water, buffer B: AcN) were filtered before use. HPLC analysis and purification were performed on a reversedphase C18 Waters Atlantis T3 column (C18-RP, $5 \mu \mathrm{m}, 6 \mathrm{~mm}, 250$ $\mathrm{mm})$. Purification of the iodinated benzoic acid was performed with method 1 (flow rate: $1 \mathrm{~mL} / \mathrm{min}$; gradient: $20 \mathrm{~min} 5 \%-95 \% \mathrm{~B} ; 25 \mathrm{~min}$ $100 \% \mathrm{~B} ; 26 \mathrm{~min} 100 \%-5 \% \mathrm{~B}$ ); quality control analysis was performed with method 1. Purification of the final product was performed on a C6 Waters Spherisorb column $(\mathrm{C} 6,5 \mu \mathrm{m}, 4.6 \mathrm{~mm} \times 250 \mathrm{~mm})$ with method 2 (flow rate: $1.5 \mathrm{~mL} / \mathrm{min}$; isocratic: $0-30 \mathrm{~min} 35 \% \mathrm{~B}$ ). SPECT imaging was performed on a NanoSPECT/CT from Mediso Medical Imaging Systems. PET imaging experiments were conducted on a Focus 120 MicroPET (Concorde Microsystems). Digital phosphor autoradiography was obtained using a Typhoon FLA 7000 laser scanner from GE Healthcare. $\gamma$-counting and biodistributions were performed using a WIZARD ${ }^{2}$ automatic $\gamma$-counter (PerkinElmer).

\section{Synthesis of ${ }^{127}$ I-PARPi}

4-(4-fluoro-3-(piperazine-1-carbonyl)benzyl)phthalazin-1(2H)-one (20 mg, $54.5 \mu \mathrm{mol}$ ) was dissolved in $1 \mathrm{~mL}$ of $\mathrm{AcN}$ and added to $10 \mathrm{mg}(41.3 \mu \mathrm{mol})$ of 3 -iodobenzoic acid, followed by $25 \mathrm{mg}$ (66 $\mu \mathrm{mol})$ of $N, N, N^{\prime}, N^{\prime}$-tetramethyl-O-(1H-benzotriazol-1-yl)uronium hexafluorophosphate (HBTU) and $20 \mu \mathrm{L}(145.5 \mu \mathrm{mol})$ of $\mathrm{Et}_{3} \mathrm{~N}$. The reaction mixture was stirred for $30 \mathrm{~min}$ and purified by HPLC (method 2) to yield the title compound $(13.4 \mathrm{mg}, 22.3 \mu \mathrm{mol}, 67 \%)$. ${ }^{1} \mathrm{H}-\mathrm{NMR}$ $\left(\mathrm{CDCl}_{3}\right) \delta=10.48(\mathrm{~s}, 1 \mathrm{H}), 8.40-8.39(\mathrm{~m}, 1 \mathrm{H}), 7.74-7.66(\mathrm{~m}, 5 \mathrm{H})$, 7.27-7.26 (d, 2H), 7.09-7.07 (d, 2H), 4.22 (s, 2H), 3.73-3.14 (m, 8H). LC-ESI-MS $(+) \mathrm{m} / \mathrm{z}=597.1\left[\mathrm{M}+\mathrm{H}^{+}\right]^{+}$. HRMS-ESI $\left[\mathrm{M}-\mathrm{H}^{+}\right]^{-} \mathrm{m} / \mathrm{z}$ calculated for $\left[\mathrm{C}_{27} \mathrm{H}_{22} \mathrm{FIN}_{4} \mathrm{O}_{3}\right]^{-}$595.0642, found 595.0640.

\section{Synthesis of ${ }^{18} \mathrm{~F}-\mathrm{PARPi}$}

${ }^{18} \mathrm{~F}$-PARPi was synthesized as described by Carney et al. (18). Briefly, a QMA cartridge containing cyclotron-produced ${ }^{18} \mathrm{~F}$-fluoride (n.c.a.) was eluted with a solution containing $9 \mathrm{mg}$ of Kryptofix [2.2.2] (4,7,13,16,21,24-hexaoxa-1,10-diazabicyclo[8.8.8]hexacosane), $0.08 \mathrm{~mL}$ of $0.15 \mathrm{M} \mathrm{K}_{2} \mathrm{CO}_{3}$, and $1.92 \mathrm{~mL}$ of AcN into a 5-mL reaction vial. Then, ethyl 4-nitrobenzoate (500 $\mu \mathrm{g}$ in $100 \mu \mathrm{L}$ of dimthyl sulfoxide [DMSO]) was added to the azeotropically dried ${ }^{18} \mathrm{~F}^{-}$and heated to $150^{\circ} \mathrm{C}$ (15 min). After this time, $50 \mu \mathrm{L}$ of $1 \mathrm{M} \mathrm{NaOH}$ were added followed by $\mathrm{HCl}(1 \mathrm{M}$, $50 \mu \mathrm{L}) 1 \mathrm{~min}$ later. Then, $2 \mathrm{mg}$ of 4-(4-fluoro-3-(piperazine-1-carbonyl) benzyl)phthalazin-1(2H)-one (in $100 \mu \mathrm{L}$ of DMSO) were added followed by $10 \mathrm{mg}$ of HBTU in $100 \mu \mathrm{L}$ of DMSO. Finally, $20 \mu \mathrm{L}$ of $\mathrm{Et}_{3} \mathrm{~N}$ were added and the reaction mixture was further diluted with $400 \mu \mathrm{L}$ of AcN and $1 \mathrm{~mL}$ of $\mathrm{H}_{2} \mathrm{O}$. The crude mixture was purified by HPLC. The decaycorrected radiochemical yield for the final step was $38.4 \pm 2.5 \%$ with a molar activity (MA) of $35.9 \pm 15.2 \mathrm{~GB} / \mu \mathrm{mol}$. The purified final compound was formulated with $10 \% \mathrm{EtOH} / 0.9 \% \mathrm{NaCl} 0.9 \%$.

\section{Synthesis of ${ }^{131}$ I-PARPi}

${ }^{131}$ I-PARPi was obtained in a manner similar to synthetic procedures reported before (14). First, ${ }^{131}$ I-NHS-benzoate was obtained by adding $N$-succinimidyl-4-(tributylstannyl) benzoate $(30 \mu \mathrm{g}, 5.9 \mu \mathrm{mol})$ in $30 \mu \mathrm{L}$ of $\mathrm{AcN}$ to a solution containing methanol $(40 \mu \mathrm{L})$, chloramine $\mathrm{T}(6 \mu \mathrm{g}, 30 \mathrm{nmol})$, acetic acid $(2 \mu \mathrm{L})$, and ${ }^{131} \mathrm{I}-\mathrm{NaI}$ in $\mathrm{NaOH}$ $0.1 \mathrm{M}$ (185-370 MBq [5-10 mCi]). After the reaction solution was driven for $15 \mathrm{~min}$ at room temperature, the reaction was purified by HLPC (method 1), and ${ }^{131}$ I-NHS-benzoate collected at $15.1 \mathrm{~min}$. The collected purified fraction was concentrated to dryness in vacuum, reconstituted in a solution of $80 \mu \mathrm{L}$ of $\mathrm{AcN}$, and added to a solution of 4-(4-fluoro-3-(piperazine-1-carbonyl)benzyl)phthalazin-1(2H)-one $(0.3 \mathrm{mg}, 0.9 \mu \mathrm{mol})$ in $20 \mu \mathrm{L}$ of DMSO and HBTU $(0.3 \mathrm{mg}, 0.8 \mu \mathrm{mol})$ in $20 \mu \mathrm{L}$ of DMSO; $10 \mu \mathrm{L}$ of 2,6-lutidine were further added and the reaction mixture stirred in an Eppendorf ThermoMixer overnight at $65^{\circ} \mathrm{C}(500 \mathrm{rpm})$. The following day, the reaction mixture was injected and purified by HPLC (method 2) and the final ${ }^{131}$ I-PARPi was collected at room temperature for $13.1 \mathrm{~min}$ (radiochemical yield $>70 \%$; radiochemical purity $>99 \%$ ) and concentrated to dryness under vacuum. ${ }^{131}$ I-PARPi was formulated with $30 \%$ PEG300/70\% saline $(0.9 \% \mathrm{NaCl})$ for both in vitro and in vivo assays $(\mathrm{MA}=1.5 \pm 0.3$ $\mathrm{GBq} / \mu \mathrm{mol})$. Coelution with nonradioactive ${ }^{127} \mathrm{I}-\mathrm{PARP}$ reference compound confirmed the identity of the radiotherapeutic. 


\section{PARP Inhibitor Binding Assays}

Assay was performed by BPS Bioscience. DMSO solutions $(100 \mu \mathrm{M})$ of each inhibitor were provided at a $100 \mathrm{nM}, 50 \mathrm{nM}$, or $10 \mathrm{nM}$ concentration. Measurements for each enzyme combined with each inhibitor were performed in triplicate in accordance with the BPS assay kit protocol (19). Luminescence was measured using a BioTek Synergy 2 microplate reader. Data were reported by BPS as percentage enzyme activity. Triplicates were combined to get a mean $\pm \mathrm{SD}$, which was then grouped and plotted onto a heat map using GraphPad Prism 7 (GraphPad Software).

\section{Cell Culture}

The human glioblastoma cell line U251 MG was kindly provided by the Laboratory of Dr. Ronald Blasberg (Memorial Sloan Kettering Cancer Center [MSKCC]). Cell lines were grown in Eagle's minimal essential medium (MEM), 10\% (vol/vol) heat-inactivated fetal bovine serum, penicillin $100 \mathrm{IU}_{2}$, and streptomycin $(100 \mu \mathrm{g} / \mathrm{mL})$, purchased from the culture medium preparation facility at MSKCC.

\section{Transduction Procedure of U87 Cell Line}

U87 human glioblastoma cells were grown in MEM with 10\% fetal calf serum. The retroviral transient producer cell line H29 was maintained in Dulbecco MEM supplemented with $10 \%$ fetal calf serum, puromycin $(2 \mu \mathrm{g} / \mathrm{mL})$, and tetracycline $(1 \mu \mathrm{g} / \mathrm{mL})$, transfected with Cis-p53/tdTomato-CBRluc-Neo and incubated for $48 \mathrm{~h}$. The U87 cells were transduced with the Cis-p53/tdTomato-CBRluc-Neo later using H29-produced virus by incubating 30\% confluent tumor cell culture with retroviral vector-containing medium for $48 \mathrm{~h}$. Transduced U87-p53/tdTomato-CBRluc-Neo cells (short: U87-p53) were selected by culturing in MEM, supplemented with $10 \%$ fetal calf serum and G418 sulfate $(0.5 \mathrm{mg} / \mathrm{mL})$ (Corning, catalog no. 30-234-CR). Several fluorescence-activated cell sorting (FACS) sorting procedures were performed to enrich cells with a low background of p53 activity. Selected cells were additionally transduced with a second constitutively expressed SFG-Turquoise/rsRluc virus as described above.

\section{Cell-Internalization, Targeting Specificity, and Binding Properties}

U251 MG cells were adhered overnight in 6-well plates (1 million cells/well) and washed twice with phosphate-buffered saline (PBS) prior to addition of ${ }^{131} \mathrm{I}$-PARPi $(222 \mathrm{kBq} /$ well, $0.15 \mathrm{nmol})$ at $37^{\circ} \mathrm{C}$ for $0,15,30,45,60,120$, and $240 \mathrm{~min}$ in MEM to allow for binding. Binding specificity was challenged with a 50 -fold excess of olaparib (3.26 $\mu \mathrm{g}, 7.5 \mathrm{nmol})$. Next, at each time point, cells were first washed twice with PBS $1 \times$ and then lysed by incubation with $1 \mathrm{M} \mathrm{NaOH}$ $\left(10 \mathrm{~min}\right.$ at $\left.37^{\circ} \mathrm{C}\right)$. Finally, the resulting lysate in each well was collected and the radioactivity measured in an automated $\gamma$-counter. Targeting specificity was assessed by a competitive binding assay between ${ }^{127}$ I-PARPi and PARPi-FL. U251 MG cells (50,000 cells/ well) were plated $48 \mathrm{~h}$ before the experiment. On the day of the experiment, binding affinity was determined by coincubating the plated cells $\left(37^{\circ} \mathrm{C}, 40 \mathrm{~min}\right)$ with serial dilutions of ${ }^{127} \mathrm{I}-\mathrm{PARP}$, ranging from 0 to $10 \mu \mathrm{M}$ and $0.5 \mu \mathrm{M}$ PARPi-FL. After this time, cells were washed twice with PBS $1 \times$, fixed, and counterstained with Hoechst DNA stain. PARPi-FL uptake and blocking effects were observed and measured by confocal microscopy.

\section{Mice}

Female athymic nude CrTac:NCr-Fo mice were purchased from Taconic Laboratories at age 6-8 wk. Xenograft-bearing mice were used to determine all pharmacokinetics, the binding/imaging properties, and the treatment efficacy of the radiotherapeutic $\left(\mathrm{n}_{\text {total }}=98\right)$. During subcutaneous injections, mice were anesthetized using 2\% isoflurane gas in $2 \mathrm{~L} / \mathrm{min}$ medical air. During orthotopic injections, mice were anesthetized using a $150 \mathrm{mg} / \mathrm{kg}$ ketamine and
$15 \mathrm{mg} / \mathrm{kg}$ xylazine cocktail ( $10 \mu \mathrm{L} / \mathrm{g}$ of body weight). For intravenous injections, the lateral tail vein was used. Mice were warmed with a heat lamp and placed in a restrainer, and the tail was sterilized with alcohol pads before injection. U251 MG or U87-p53 cells were implanted subcutaneously $\left(5 \times 10^{6}\right.$ cells in $150 \mu \mathrm{L}$ of $1: 1$ PBS/Matrigel [BD Biosciences]) into the right shoulder and allowed to grow for approximately 2 wk until the tumors reached about $8 \mathrm{~mm}$ in diameter $(100 \pm 8 \mathrm{~mm})$. For orthotopic injections, U251 MG or U87-p53 cells $\left(5 \times 10^{5}\right.$ cells in $2 \mu \mathrm{L}$ of PBS $)$ were injected $2 \mathrm{~mm}$ lateral and $1 \mathrm{~mm}$ anterior to the bregma using a Stoelting Digital New Standard Stereotaxic Device and a $5-\mu \mathrm{L}$ Hamilton syringe and allowed to grow for approximately $3 \mathrm{wk}$. All mouse experiments were performed in accordance with protocols approved by the Institutional Animal Care and Use Committee of MSKCC and followed National Institutes of Health guidelines for animal welfare.

\section{SPECT/CT}

SPECT/CT scans were obtained using a small-animal NanoSPECT/ CT from Mediso Medical Imaging Systems. For subcutaneous U251 MG xenografts, ${ }^{131} \mathrm{I}$-PARPi $(11.1 \mathrm{MBq}, 1.5 \mathrm{GBq} / \mu \mathrm{mol}$ in $20 \mu \mathrm{L}$ of $30 \%$ PEG300 in $0.9 \%$ sterile saline) was administered intratumorally $(n=3)$. At 0 and $6 \mathrm{~h}$ after injection, the mice were anesthetized with $1.5 \%-2.0 \%$ isoflurane (Baxter Healthcare) at $2 \mathrm{~mL} / \mathrm{min}$ in oxygen, and SPECT/CT data were acquired for $20 \mathrm{~min}$. For orthotopic U251 MG tumor-bearing mice, ${ }^{131} \mathrm{I}$-PARPi $(1.9 \mathrm{MBq}, 1.5 \mathrm{GBq} / \mu \mathrm{mol}$ in $5 \mu \mathrm{L}$ of $30 \%$ PEG300 in $0.9 \%$ sterile saline) was injected intracranially using the same coordinates as for tumor cell injection $(2 \mathrm{~mm}$ lateral and $1 \mathrm{~mm}$ anterior to the bregma using a Stoelting Digital New Standard Stereotaxic Device and a 5- $\mu$ L Hamilton syringe). At $24 \mathrm{~h}$ after injection, the mice were anesthetized with $1.5 \%-2.0 \%$ isoflurane (Baxter Healthcare) at $2 \mathrm{~mL} / \mathrm{min}$ in oxygen, and SPECT/CT data were collected for $20 \mathrm{~min}$ in the head region.

\section{Autoradiography and Hematoxylin and Eosin Staining}

Twenty-four hours after intratumoral injection of ${ }^{131}$ I-PARPi or olaparib/ ${ }^{131}$ I-PARPi in U251 MG subcutaneous xenograft-bearing mice, mice were sacrificed and tumors were harvested. The collected organs were embedded in Tissue-Tek O.C.T. compound (Sakura Finetek), flash-frozen in liquid nitrogen, and cut into 20$\mu \mathrm{m}$ sections using a Vibratome UltraPro 5000 Cryostat (Vibratome). A storage phosphor autoradiography plate (BAS-MS2325; Fiji Photo Film) was exposed to the tissue slices overnight at $-20^{\circ} \mathrm{C}$ and read the following day. Relative count intensity of the sections in each image was quantified using ImageJ $1.47 \mathrm{u}$ processing software (NIH). Tumor-to-muscle and brain-to-muscle ratios were calculated using Prism 6.0c (GraphPad Software). Sections were subsequently subjected to hematoxylin and eosin staining for morphologic evaluation of tissue pathology.

\section{PET/CT Imaging}

Small-animal PET imaging data were recorded on a Focus 120 MicroPET (Concorde Microsystems) and reconstructed using AsiPro VM MicroPET Analysis software (Siemens Medical Solutions). ${ }^{18} \mathrm{~F}$-PARPi $(5.55 \mathrm{MBq}, 35.89 \pm 15.17 \mathrm{GBq} / \mu \mathrm{mol}$ in $300 \mu \mathrm{L}, 10 \%$ $\mathrm{EtOH}$ in $0.9 \%$ sterile saline) alone or following an intratumoral injection of ${ }^{127} \mathrm{I}$-PARPi $(10 \mu \mathrm{g}, 16.5 \mu \mathrm{mol}, 20 \mu \mathrm{L}, 30 \%$ PEG300/70\% $\mathrm{NaCl} 0.9 \% 6 \mathrm{~h}$ before ${ }^{18} \mathrm{~F}$-PARPi injection) was injected into U251 MG tumor-bearing mice $(n=3)$ via the tail vein. At $2 \mathrm{~h}$ after injection, the mice were anesthetized with $1.5 \%-2.0 \%$ isoflurane (Baxter Healthcare) at $2 \mathrm{~mL} / \mathrm{min}$ in oxygen, and PET/CT imaging was performed over $15 \mathrm{~min}$. Images were analyzed using AsiPro VM software (Concorde Microsystems). Then, mice were sacrificed and key 
organs harvested for quantification and autoradiography/hematoxylin and eosin staining.

\section{Ex Vivo Biodistribution and Dosimetry (Subcutaneous Model)}

Biodistribution studies were performed in subcutaneous U251 MG xenograft-bearing athymic nude mice $(n=44,11$ randomized cohorts, 4 mice per cohort). Mice were divided into 2 groups (blocked and unblocked), and ${ }^{131} \mathrm{I}-\mathrm{PARPi}(0.56 \mathrm{MBq}, 1.5 \mathrm{GBq} /$ $\mu \mathrm{mol}$ in $20 \mu \mathrm{L}, 30 \%$ PEG300 in $0.9 \%$ sterile saline) was administered intratumorally. The blocked group was preinjected $30 \mathrm{~min}$ before ${ }^{131} \mathrm{I}$-PARPi with olaparib $(8.15 \mu \mathrm{g}, 18 \mathrm{nmol}$ in $100 \mu \mathrm{L}$, $30 \% \mathrm{PEG} 300 / 70 \% \mathrm{NaCl} 0.9 \%$ ). Mice were sacrificed by $\mathrm{CO}_{2}$ asphyxiation at 1, 3, 6, 12, 24, 48, 72, and $96 \mathrm{~h}$ after injection (for ${ }^{131}$ I-PARPi-only groups) and at 1,6 , and $24 \mathrm{~h}$ after injections (for blocking experiments groups), and major organs were collected, weighed, and counted in a WIZARD $^{2}$ automatic $\gamma$-counter (PerkinElmer). The ${ }^{131}$ I-PARPi uptake was expressed as a percentage of injected dose per gram (\%ID/ g) using the following formula: [(activity in the target organ/g of tissue)/injected dose] $\times 100 \%$ (Supplemental Figs. 5 and 6; supplemental materials are available at http://jnm.snmjournals.org). The average of the 4 organ specimens from each time point was fitted to a biexponential function. The best fit for most organs comprised a 2phase exponential clearance model. For 2 organs (the thyroid and large intestine), there was measured uptake over the first $12 \mathrm{~h}$, and therefore these organs were fitted to an uptake and clearance exponential model. Cumulative uptake was calculated from the areas under the tumor uptake curves. Absorbed doses to tumor and all normal organs were estimated assuming absorbed fractions of 1 for the $\beta$-emissions of ${ }^{131} \mathrm{I}$ and 0 for the penetrating photon emissions and using an equilibrium dose constant of $0.111 \mathrm{~g} \mathrm{~Gy} / \mathrm{MBq}$ h $(0.405 \mathrm{~g} \mathrm{cGy} / \mu \mathrm{Ci}$ h). The ratio of tumor to normal organ dose exceeded 20 for all organs except the thyroid (Supplemental Fig. 4). The high dose to the thyroid gland was a likely consequence of the deiodination followed by uptake and then slow clearance (evidence of trapping in the thyroid tissue) (Supplemental Fig. 7).

\section{Bioluminescence}

Bioluminescence studies were performed in subcutaneous U87p53 xenograft-bearing mice ( $n=8,4$ mice per cohort). Mice were divided into 2 groups (treated and untreated). For treatment, mice were administered ${ }^{131} \mathrm{I}$-PARPi $(14.8 \mathrm{MBq}, 1.5 \pm 0.3 \mathrm{GBq} / \mu \mathrm{mol}$ in $20 \mu \mathrm{L}, 30 \%$ PEG300 in $0.9 \%$ sterile saline) intratumorally. The untreated group received an intratumoral injection of a 30\% PEG300 solution in $0.9 \%$ sterile saline $(20 \mu \mathrm{L})$. Imaging was performed on an IVIS Spectrum preclinical in vivo imaging system from PerkinElmer the day before treatment (baseline) and on day 0, 1, and 2 after treatment. For detection of the constitutively expressed SFG-Turquoise/rsRluc reporter, mice were given a retroorbital injection of $10 \mu \mathrm{g}$ of coelenterazine $\mathrm{h}$ ((2-(4-dehydroxy)coelenterazine from Biotium) dissolved in $5 \%$ ethanol in $0.9 \%$ sterile saline solution and imaged within $22 \mathrm{~s}$ of injections. For detection of p53 activation (Cis-p53/tdTomato-CBRluc-Neo), mice were imaged 20 min after an intraperitoneal injection of $2 \mathrm{mg}$ of luciferin $/ 100 \mu \mathrm{L}$ of sterile $0.9 \%$ $\mathrm{NaCl}$ solution.

\section{Therapeutic Efficacy}

Therapeutic efficacy studies were performed in subcutaneous U87-p53 xenograft-bearing mice $(n=29,3$ randomized groups, 9-10 mice per cohort). Mice were divided into 3 cohorts (vehicle, nonradioactive/cold ${ }^{127} \mathrm{I}$-PARPi, and $3 \times 14.8 \mathrm{MBq}$ of ${ }^{131} \mathrm{I}$-PARPi). The cold group received mass equivalent doses $(6 \mu \mathrm{g})$ of ${ }^{127} \mathrm{I}$-PARPi. Vehicle, ${ }^{127} \mathrm{I}$-PARPi, and $3 \times{ }^{131}$ I-PARPi were administered on day 0,3 , and 6 via intratumoral injections. Body weights were recorded every Monday, Wednesday, and Friday until tumor burden exceeded the endpoint of $800 \mathrm{~mm}$.
Additional supporting tables and figures can be found in the supplemental materials. All animal experiments were performed in accordance with protocols approved by the Institutional Animal Care and Use Committee and followed the National Institutes of Health guidelines for animal welfare.

\section{Orthotopic CED Model}

Mice received intracranial injections of U87-p53 cells $\left(5 \times 10^{4}\right.$ cells in $1.5 \mu \mathrm{L}$ of PBS). After $3 \mathrm{wk}$, bioluminescence imaging was used to determine presence of tumor. Then, we surgically implanted an ALZET Osmotic Pump into the mice, which slowly delivered an infusion into the brain using the same coordinates as for the tumor cell injection. Both healthy and diseased mice received an ALZET Osmotic Pump (Model 1003D) with Brain Infusion Kit 3 containing ${ }^{131} \mathrm{I}-$ PARPi $(40.7 \pm 11.1 \mathrm{MBq}$ in $101 \pm 8 \mu \mathrm{L} 30 \%$ PEG300/70\% NaCl $0.9 \%$ ). Pumps were weighed before and after filling to calculate added volume, as per the manufacturer's instruction. After filling pumps and assembling catheter and cannula, infusion kits were incubated in $0.9 \%$ saline at $37^{\circ} \mathrm{C}$ overnight to ensure flow was initiated $(1.0 \mu \mathrm{L} / \mathrm{h}$ $[ \pm 0.15 \mu \mathrm{L} / \mathrm{h}])$ and air bubbles were removed. Immediately before implantation, pumps were measured in dose calibrators to measure activity in pumps at time of surgery. SPECT/CT images were acquired 1 $\mathrm{h}$ after surgery and again at $12 \mathrm{~h}$. Mice were imaged every $24 \mathrm{~h}$ for 6 days. After the fifth day, the pumps were surgically removed, and the remaining dose was measured in dose calibrators to ensure payload had been delivered. The image volumes were segmented using a combination of manual and semiautomatic segmentation techniques in 3D Slicer, version 4.6 (www.slicer.org) for quantification of absolute activity in different organs at each time point. The presence of activity levels above background in the SPECT images was not observed except in the brain/tumor and the osmotic pump.

\section{Dosimetry (CED)}

Source/Target Definition. The popular Digimouse atlas (20) was used as a digital phantom for dosimetry calculations. The atlas was adapted in the open-source 3-dimensional modeling software Blender to incorporate models of the osmotic pump, tumor, and thyroid tissues. The adapted model was tetrahedralized via the Delaunay method in the mesh generation software Tetgen to construct a 3-dimensional finite element mesh suitable for implementation in the Particle and Heavy Ion Transport Simulation (PHITS) Monte Carlo code (21). Material attributes for each region were assigned as soft tissue, lung, or bone using standard International Commission on Radiological Protection reference data for atomic composition and density (www. physics.nist.gov).

Calculation of Injected Activity. The rate of injection of ${ }^{131}$ I activity into the tumor is:

$$
\frac{d A_{i}}{d t}=-\frac{d A_{p}}{d t}-\lambda A_{p}
$$

where, $A_{i}=$ injected activity $(\mu \mathrm{Ci}), A_{p}=$ activity in pump $(\mu \mathrm{Ci})$, $\lambda=$ radioactive decay constant $\left(\mathrm{h}^{-1}\right)$, and $t=$ time after implantation (h). Given $A_{p}=A_{O}$ at $t=0$, the cumulative injected activity is obtained following solution of Equation 1 using an integrating factor or transform methods:

$$
A_{i}=A_{0}-\int_{0}^{t} A_{p} d t-A_{p}
$$

where, $A_{0}=$ initially implanted activity $(\mu \mathrm{Ci}) . A_{0}$ was determined by measuring the activity present in the pump in a dose calibrator immediately prior to implantation. $A_{p}$ at different time points was 
determined from SPECT imaging volume-of-interest analysis. The resulting time-activity curve was fit as a 1-phase exponential decay; the fitted curve was then implemented for calculation of the injected activity via Equation 2 (Supplemental Fig. 10E).

Monte Carlo Simulation. PHITS was used to generate decay events in each source via rejection sampling; betas and photons were simulated using a spectrum of beta energies obtained from the RADAR database (www.doseinfo-radar.com) for ${ }^{131} \mathrm{I}$, gamma and $\mathrm{x}$-ray spectra were obtained from the International Atomic Energy Agency Nuclear Data Center (www-nds.iaea.org), and a 3-keV energy cutoff was used. The activity in each source tissue is assumed to be distributed uniformly and is assigned a random initial direction vector. Total events $\left(1-5 \times 10^{6}\right)$ were simulated, resulting in less than $10 \%$ relative error in energy deposit in relevant target tissues. Absorbed doses were output either in units of Gy or normalized to the injected activity calculated using Equation 2 (Gy/uCi). Three-dimensional dose maps were rendered in Paraview (Sandia National Laboratory, Kitware Inc., Los Alamos National Laboratory).

\section{RESULTS}

\section{Synthesis, Specificity, and In Vitro Performance of 131I-PARPi}

Several research groups have synthesized PARP-targeted radiotracers in the past; some of which were attached to radioiodine $(14,22-24)$. We showed that one of these tracers, ${ }^{131}$ I-PARPi (14), has a high affinity with a concentration at which $50 \%$ of binding has been inhibited $\left(\mathrm{IC}_{50}\right)$ in the nanomolar range $(11 \pm 3 \mathrm{nM})$ and a $\log \mathrm{P}_{\mathrm{CHI}}$ of 2.3. ${ }^{131} \mathrm{I}$-PARPi was synthesized with a final molar activity of $1.5 \mathrm{GBq} / \mu \mathrm{mol}$. Radiochemical purity was $99.1 \% \pm$ $0.9 \%$ for all prepared compounds. Pharmacologically, ${ }^{131}$ I-PARPi behaves in a manner similar to the Food and Drug Administrationapproved PARP inhibitor olaparib (Fig. 1; Supplemental Fig. 1; (25)). We tested the inhibitory activity of ${ }^{127}$ I-PARPi on a panel of 12 PARP enzymes, including tankyrase 1 and tankyrase 2 (Fig. 1B). We found near-perfect overlap with literature-known values for ${ }^{18} \mathrm{~F}$-PARPi, PARPi-FL, and olaparib at $100 \mathrm{nM}$ (12). ${ }^{18} \mathrm{~F}$-PARPi is a PARP-targeted PET imaging agent with an $\mathrm{IC}_{50}$ of $3 \mathrm{nM}$ (Supplemental Fig. 1) (18). PARPi-FL is a fluorescent
PARP inhibitor with an $\mathrm{IC}_{50}$ value of $12.2 \pm 1.1$ (17). The 2 dominant targets for ${ }^{127}$ I-PARPi were found to be PARP-1 and PARP-2, just as for ${ }^{18} \mathrm{~F}-\mathrm{PARPi}$, PARPi-FL, and olaparib (26).

The in vitro binding specificity and internalization of ${ }^{131}$ I-PARPi was shown by incubating U251-MG cells with ${ }^{131} \mathrm{I}$-PARPi in the presence and absence of $150 \mu \mathrm{L}$ of $6.67 \mu \mathrm{g} / \mu \mathrm{L}$ olaparib solution suspended in $25 \mu \mathrm{L}$ of DMSO, $25 \mu \mathrm{L}$ of polyethylene glycol (PEG), and $100 \mu \mathrm{L}$ saline (Fig. 1C). In both cases, binding reached a plateau at approximately $50 \mathrm{~min}$ and was 10 -fold higher in cells exposed to only ${ }^{131}$ I-PARPi than in cells coincubated with olaparib. Additionally, we showed that the radiotherapeutic binds to its intended target within the nucleus by incubating U251-MG cells with serial dilutions of its nonradioactive counterpart, ${ }^{127} \mathrm{I}-\mathrm{PARP}$, in the presence of $0.5 \mu \mathrm{M}$ PARPi-FL. Overall, uptake of PARPi-FL retained in the nucleus at a concentration of $0.5 \mu \mathrm{M}$ could be suppressed by addition of a 20 -fold excess of ${ }^{127}$ I-PARPi (Supplemental Fig. 2B).

\section{Intratumoral Injection and Tissue Perfusion of ${ }^{131}$ I-PARPi}

Intratumoral injections were implemented with a clinical CED model in mind, as well as for the favorable biodistribution (Fig. 2). To determine how intratumorally injected ${ }^{131} \mathrm{I}-\mathrm{PARPi} /{ }^{127} \mathrm{I}-\mathrm{PARPi}$ distributes throughout a tumor, we designed an in vivo competitive binding specificity experiment with ${ }^{18} \mathrm{~F}-\mathrm{PARP}$, a previously described PET imaging agent for PARP-1. One cohort of animals was intratumorally injected with ${ }^{127} \mathrm{I}-\mathrm{PARPi}$, followed by ${ }^{18} \mathrm{~F}$ PARPi 30 min later. The second cohort was injected with ${ }^{18} \mathrm{~F}$ PARPi only (Fig. 2A). We found that intratumoral injection of ${ }^{127}$ I-PARPi suppressed uptake of ${ }^{18} \mathrm{~F}$-PARPi, indicating that the small molecule occupies ${ }^{18} \mathrm{~F}$-PARPi binding sites (Figs. $2 \mathrm{~B}$ and $2 \mathrm{C})$, similar to what we showed in ${ }^{18} \mathrm{~F}-\mathrm{PARPi} /$ olaparib blocking experiments previously (18). Ex vivo quantification at $1 \mathrm{~h}$ after injection of ${ }^{18} \mathrm{~F}$-PARPi demonstrated that uptake dropped by 1.7 $\pm 0.1 \% \mathrm{ID} / \mathrm{g}(1.9 \pm 0.3$ and $0.18 \pm 0.07 \% \mathrm{ID} / \mathrm{g}, P<0.0001)$ between mice without and with intratumoral injection of ${ }^{127} \mathrm{I}-$ PARPi (Fig. 2C). When replicated with olaparib and ${ }^{131} \mathrm{I}-\mathrm{PARP}$, brain uptake was low between mice without and with intravenous injection of olaparib $(0.056 \pm 0.020$ and $0.061 \pm 0.026 \% \mathrm{ID} / \mathrm{g}$, respectively) (Supplemental Figs. 3 and 4). Using autoradiography, we found heterogeneity in the intratumoral distribution of ${ }^{131} \mathrm{I}$-PARPi (Supplemental Fig. 5A). Overall quantification, however, corroborated the ${ }^{18} \mathrm{~F}-$ PARPi PET experiments (Fig. 2), and ratios between animals receiving only ${ }^{131} \mathrm{I}$-PARPi versus animals receiving a preinjection of olaparib were $7.8(144.3 \pm 32.7 \mathrm{AU}$ and $18.5 \pm 1.2 \mathrm{AU}, P<0.0001$ for mice without and with preinjection of olaparib) (Supplemental Fig. 5B).

\section{I-PARPi Pharmacokinetics and Dosimetry in Glioblastoma}

CED delivery of therapeutics to the brain relies on a drug's fast perfusion, tissue permeability, and clearance from areas not expressing the targeted biomarker. To simulate this setting, we tested retention of ${ }^{131}$ I-PARPi in 2 cohorts of U251-MG subcutaneous xenografts. In 1 cohort, PARP was made unable
FIGURE 1. (A) In vitro studies of PARP inhibitors. Structure of ${ }^{131}$ I-PARPi (14). (B) Heat map of binding characteristics of synthetic inhibitors (at $100 \mathrm{nM}$ ) to PARP family enzymes in percentage enzyme inhibition. (C) Cellular uptake of ${ }^{131}$ I-PARPi alone and blocked with mass excess dose of olaparib. 


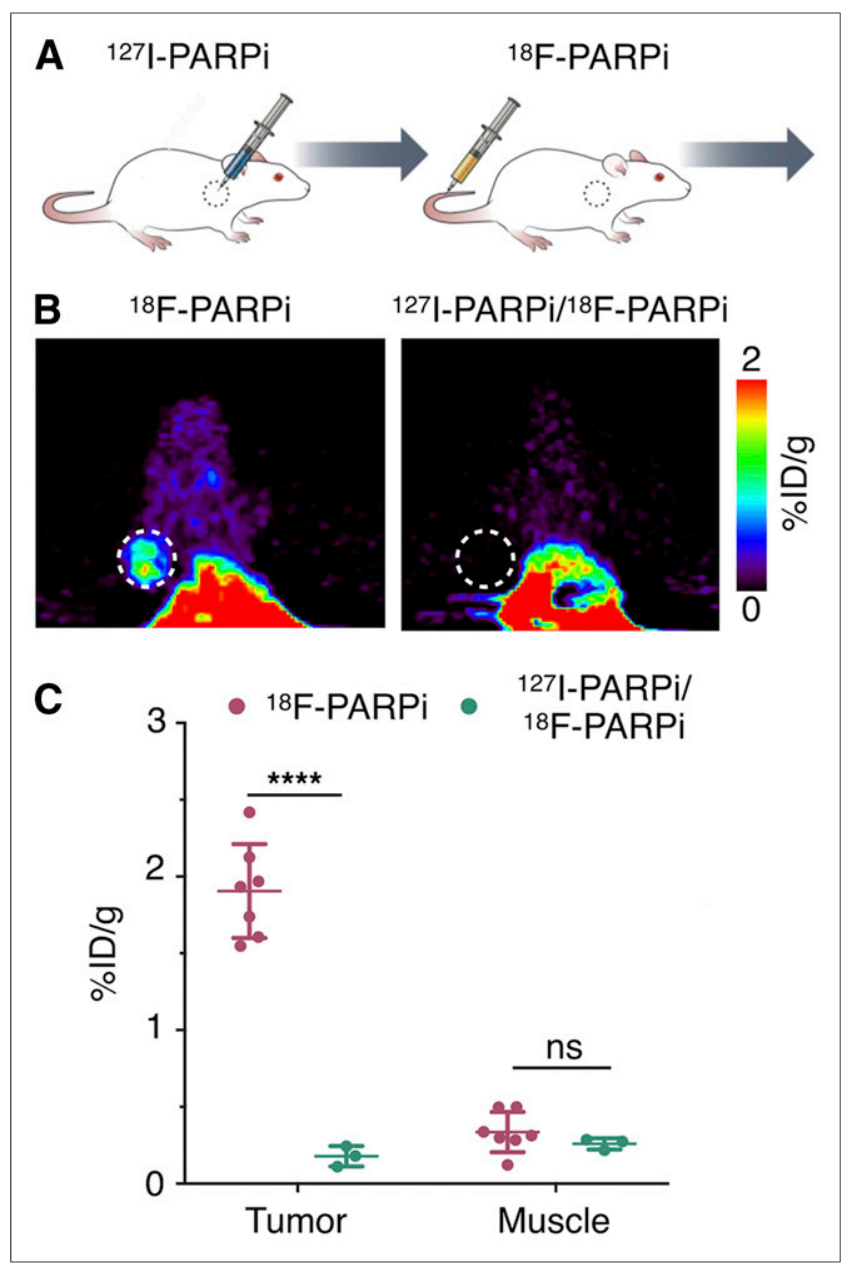

FIGURE 2. ${ }^{131}$ I-PARPi binding specificity. (A) Experimental outline of immuno-PET/CT imaging after injection of nonradioactive version of ${ }^{131}$ I-PARPi (127I-PARPi). (B) ${ }^{18} \mathrm{~F}$-PARPi PET tracer injected intravenously 30 min before intratumoral injection of ${ }^{127}$ I-PARPi. PET scan of mice $2 \mathrm{~h}$ after receiving ${ }^{18} \mathrm{~F}$-PARPi with and without ${ }^{127}$ I-PARPi before injection. (C) Ex vivo biodistribution of ${ }^{18} \mathrm{~F}$-PARPi $1 \mathrm{~h}$ after injection in selected tissue. ${ }^{\star \star \star \star} P<0.0001$. Error bars represent SD.

to retain the radiotherapeutic through intravenous preinjection of olaparib. NanoSPECT/CT showed that at $6 \mathrm{~h}$ after intratumoral injection of ${ }^{131}$ I-PARPi (11.1 MBq, $20 \mu \mathrm{L}, 30 \%$ PEG/0.9\% $\mathrm{NaCl}$ solution), the radiotherapeutic was retained in tumors not previously injected with olaparib, whereas it had almost completely cleared from the blocked group (Fig. 3A). This observation was further corroborated by biodistribution experiments with endpoints (each time point $n=4$ ) between 0 and $96 \mathrm{~h}$ after injection (Figs. 3B and 3C; Supplemental Figs. 3, 4, and 6). On the basis of these data, we calculated the activity and dose retained in different tissues (Supplemental Fig. 7). Intuitively, the highest dose was delivered to tumor tissue, with a calculated absorbed dose of $134.1 \mathrm{cGy} / \mathrm{MBq}$. This was followed by the thyroid (68.6 cGy/MBq), which metabolizes and stores iodine (Supplemental Fig. 6; (27)). Besides PARP expression in the thyroid, storage of metabolized iodine is likely one reason for this comparatively high calculated dose. This is supported by the finding that in this setting, physical decay was observed as the only mode of clearance. However, moving forward, thyroid uptake can efficiently be blocked by administration of nonradioactive iodide (28).

\section{Bioluminescence and p53 Response}

We designed and transduced a reporter cell line responding to p53 activation, a marker of cell death and radiation damage. The cells coexpress $\mathrm{p} 53 /$ luciferase, together with constitutively expressing SFGTurquoise/rsRluc. These bioluminescent reporters allow p53 expression to be imaged after injection of luciferase, and cell density to be imaged after injection of coelenterazine. Using this setup, we used bioluminescence imaging to determine $\mathrm{p} 53$ expression in relation to cell density (29). To use this experimental setup for testing whether ${ }^{131}$ I-PARPi retention is sufficient for inducing cell death, mice bearing subcutaneous transfected U87-p53 xenografts were injected with $16.3 \pm 1.5 \mathrm{MBq}$ of ${ }^{131}$ I-PARPi or vehicle (Supplemental Fig. 8). Dual reporter bioluminescence was measured $1 \mathrm{~d}$ before, on the day of, and 24 and $48 \mathrm{~h}$ after ${ }^{131}$ I-PARPi injection. For the control groups, no statistical differences were observed for any time points, suggesting that vehicle alone did not induce cell death (Supplemental Fig. 8). For mice injected with ${ }^{131}$ I-PARPi, however, a statistically significant increase in p53 signal was observed $24 \mathrm{~h}$ after administration of ${ }^{131} \mathrm{I}-$ PARPi, which persisted at $48 \mathrm{~h}$ after injection $(P<0.005$ after $24 \mathrm{~h}$ and $P<0.05$ after $48 \mathrm{~h}$ when compared with day 0$)$.

\section{Therapeutic Efficacy of ${ }^{131}$ I-PARPi in Mouse Model of Glioblastoma}

We next sought to determine the therapeutic efficacy of ${ }^{131} \mathrm{I}$ PARPi in a subcutaneous xenograft model of transduced U87-p53. For this purpose, tumor-bearing mice were randomly assigned to 3 different cohorts ( $n=9-10$ per cohort): vehicle (30\% PEG-300/ PBS), nonradioactive ${ }^{127} \mathrm{I}-\mathrm{PARPi}(5.9 \mu \mathrm{g}, 9.9 \mathrm{nmol})$, or fractionated doses of ${ }^{131}$ I-PARPi $(3 \times 14.8 \mathrm{MBq}, 3 \times 9.9 \mathrm{nmol})$. For the fractionated-dose cohort, vehicle and cold ${ }^{127}$ I-PARPi intratumoral injections were performed on days 0,3 , and 6 . Body weights and tumor volumes were recorded 3 times per week for the length of the study (Supplemental Fig. 9B); neither cohort experienced statistically significant weight loss, confirming that the administered activity did not lead to systemic toxicity (Supplemental Fig. 9B). We performed 2-sample $t$ tests to compare tumor growth curves under dependent right censoring to account for sacrifices due to ethical guidelines and applied a correction for the multiple pairwise group comparisons (Supplemental Fig. 9D) (30). No statistical difference of tumor volume was observed between the vehicle cohort and the ${ }^{127}$ I-PARPi cohort. The fractionated-dose treatment led to a statistically significant reduction in tumor volume compared with the vehicle group $(P<0.005)$. This is also reflected in the tumor growth rates, which were not different at the beginning of the study (week 0) but showed reduced growth rates in the 2 radiotherapy groups at week 2 compared with the control group (Fig. 4A). When comparing survival of mice, statistical significance was found for the treatment group versus the vehicle $(P<0.005)$. No statistical difference was found between the vehicle group and cold ${ }^{127}$ I-PARPi. Mice receiving fractionated doses had the longest median survival (29 d). Median survival for the PBS group and cold ${ }^{127}$ I-PARPi group was 22 and 20 d, respectively (Fig. 4B).

\section{Orthotopic Model}

Simulating a potential clinical treatment scenario, we tested the delivery of ${ }^{131}$ I-PARPi in an orthotopic U87-p53 mouse model of glioblastoma paired with an implantable osmotic pump and 


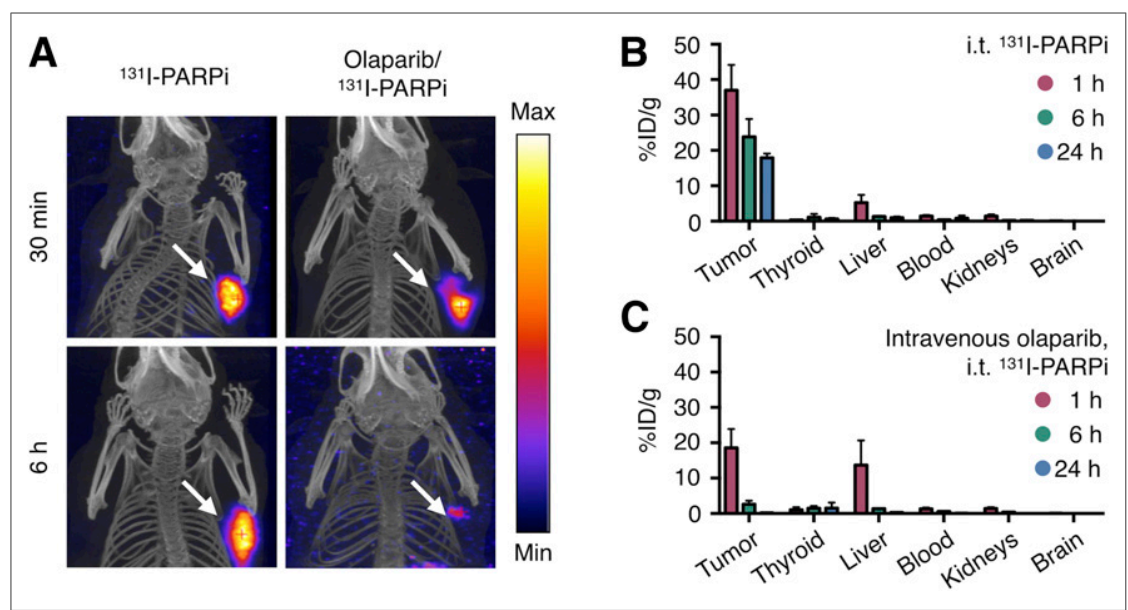

FIGURE 3. In vivo binding, biodistribution, and pharmacokinetics. (A) SPECT/CT images of subcutaneously U87-p53-xenografted mice intratumorally injected with $(11.1 \mathrm{MBq}){ }^{131} \mathrm{I}$-PARPi at time of injection and $6 \mathrm{~h}$ after injection. (B) Blocked mice received a systemic blocking dose of Olaparib $1 \mathrm{~h}$ before ${ }^{131}$ I-PARPi. ${ }^{131}$ I-PARPi (14.8 MBq) biodistribution in key organs after intratumoral injection at 1,6 , and $24 \mathrm{~h}$ ( $n=4$ per time point). (C) ${ }^{131} \mathrm{I}-\mathrm{PARPi}$ biodistribution in key organs at 1,6 , and $24 \mathrm{~h}$ postintratumoral injection after systemic blocking via intravenous injection of mass excess olaparib ( $n=4$ per time point).

cannula (Fig. 5A). The osmotic pump mimicked slow, CED-type delivery of our radiotherapeutic, and we compared the retention of ${ }^{131}$ I-PARPi in tumor-bearing versus healthy mice (Supplemental Fig. 10). On the basis of calculations of the administered dose from SPECT-derived pump activity concentrations versus time, it was shown that administration of $30.7 \mathrm{MBq}(49.2 \mathrm{MBq}$ initially implanted) resulted in the highest doses in the brain/tumor itself (30.1622 \pm $0.1946 \mathrm{cGy} / \mathrm{MBq}$ ID), induced overwhelmingly by tumor-bound ${ }^{131}$ I-PARPi $(27.4405 \pm 0.03784 \mathrm{cGy} / \mathrm{MBq}$ ID) versus photon dose from the pump contents $(2.0768 \pm 0.100 \mathrm{cGy} / \mathrm{MBq}$ ID; Supplemental Fig. 5). Other organs, affected primarily by $\gamma$-radiation from the pump contents, received comparatively small doses (Fig. 5B). Only the left lung, which was in direct proximity to the osmotic pump activity reservoir, received a somewhat elevated dose

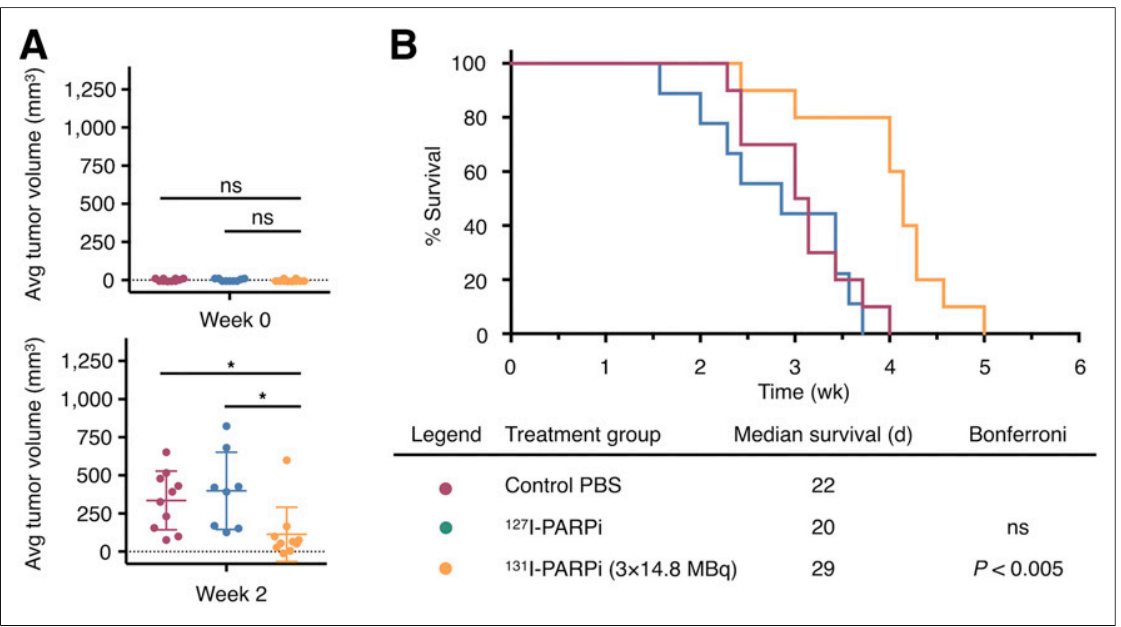

FIGURE 4. In vivo therapeutic effect of ${ }^{131}$ I-PARPi therapy in subcutaneous mouse model. (A) Difference of tumor growth percentage at beginning of study and at 2 wk. (B) Kaplan-Meier plot and table of treatment groups with median survival. Nonparametric Student $t$ test was used to calculate statistics for A. ${ }^{\star} P<0.05$. Error bars represent SD. $P$ values were calculated by MantelCox test for $\mathrm{B}, P=0.0001$.
(11.08 cGy/MBq injected dose). Experimental findings corroborated these models, as strong, durable retention of ${ }^{131}$ I-PARPi was observed in tumor-bearing mice, whereas healthy brain tissue retained the radiotherapeutic to a much lower degree (Fig. 5C); after $6 \mathrm{~d}, 9.1$ Gy were delivered to the brains of tumor-bearing mice, whereas healthy mice retained less than $1 \mathrm{~Gy}$.

\section{DISCUSSION}

There is a critical need for more and better therapeutic options for patients with glioblastoma. The past few decades have seen only modest improvements in treatment outcomes (31). Both PARP inhibition therapy and targeted radionuclide therapy are 2 new treatment approaches that have independently gained widespread attention; both types of therapy have led to clinical trials, which are currently ongoing (32). Here, we fuse these 2 approaches and use PARP as a target for a newly developed targeted radiotherapeutic. In this study, we sought to demonstrate a CED approach to delivering the radiotherapeutic molecule ${ }^{131}$ I-PARPi in orthotopic glioblastoma models.

In vitro and in vivo studies have shown that ${ }^{131} \mathrm{I}$-PARPi is a potent PARP inhibitor, and that introduction of radioiodine did not perturb binding specificity. This was also confirmed in a 12member cross-family binding assay showing that ${ }^{131}$ I-PARPi possesses a binding profile akin to olaparib and other olaparib-based imaging agents (Fig. 1). PET imaging with ${ }^{18} \mathrm{~F}$-PARPi demonstrated that intratumoral administration of the cold iodinated analog ${ }^{127} \mathrm{I}-$ PARPi perfused efficiently throughout the tumor (Fig. 2), confirming that the tissue permeability of the small molecule is high, similar to what we found for PARPi-FL (33). We also measured the pharmacokinetics of the compound when administered intratumorally and observed strong retention of activity in the tumor if PARP is available, but only very little when PARP binding sites have been saturated with olaparib. Intuitively, the deposited dose is a function of the small molecule's tissue half-life, and the difference between the 2 is a function of target selectivity (Fig. 3). These data were used in dosimetric analysis, which showed that the tumor received $134.1 \mathrm{cGy} / \mathrm{MBq}$ while the major clearance organs, the thyroid and liver, received doses of 68.4 and 6.2 cGy/MBq, respectively. Using a fluorescently transfected cell line capable of reporting p53 activation, we saw a statistically significant increase in p53 activation in subcutaneous mouse models treated with ${ }^{131}$ I-PARPi (Supplemental Fig. 7). This activation serves as a marker of radiation damage to cells, further verifying the therapeutic potential of ${ }^{131} \mathrm{I}-$ PARPi. We then conducted a survival study with the subcutaneous mouse model that 


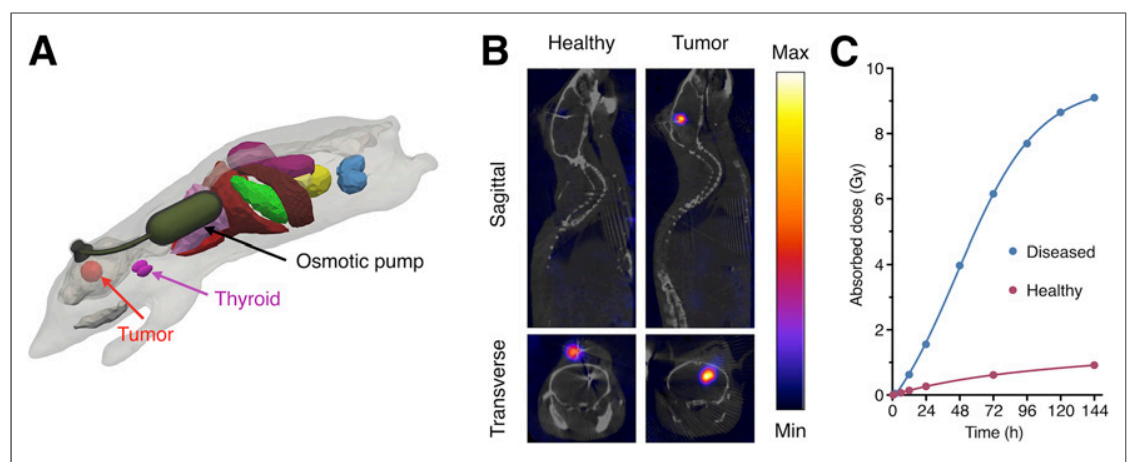

FIGURE 5. In vivo CED in orthotopic glioblastoma mouse model. (A) Three-dimensional model of orthotopic glioblastoma mouse model with CED-mimicking osmotic pump. (B) SPECT/CT of orthotopic U87-p53 tumors during osmotic pump treatment of ${ }^{131}$ I-PARPi at 72 h. (C) Calculated absorbed dose to brain* during treatment. *Brain and tumor have been considered together as a single organ in organ-level dose calculation.

showed a significant reduction in tumor growth and improvement in median survival when ${ }^{131}$ I-PARPi was administered intratumorally as either a single dose of $14.8 \mathrm{MBq}$ or as a fractionated dose of $3 \times$ 14.8 MBq (Fig. 4). Low toxicity, as a function of the mouse's body weight, was observed throughout this study.

${ }^{131}$ I-PARPi represents a theranostic pair in itself, insofar as it might be used for SPECT imaging when delivered systemically in addition to its therapeutic function when delivered intratumorally. However, in addition to SPECT, the ${ }^{18} \mathrm{~F}$-labeled analog ${ }^{18}$ F-PARPi (18) can be used for PET imaging, which may be more suitable for patient stratification and treatment monitoring. Additionally, the fluorescently labeled analog PARPi-FL (16) might be used for fluorescence-guided resections.

A likely clinical scenario of ${ }^{131}$ I-PARPi treatment involves the radiotherapeutics' administration as a CED agent, similar to what has been shown previously for antibodies (7). ${ }^{131}$ I-PARPi has structural similarity to olaparib, which features a cyclopropamide instead of the ${ }^{131}$ I-labeled meta-iodobenzamide. Olaparib is a substrate of phosphatidylglycerolphosphate synthase 1 , a multidrug efflux transporter $(34,35)$. PGP might therefore be important in mediating ${ }^{131} \mathrm{I}-$ PARPi uptake in contrast-enhancing and non-contrast-enhancing regions of the brain, affecting both uptake and clearance of drug, but potentially also improving selective uptake. To mimic a potential clinical CED scenario, mice bearing orthotopic glioblastoma xenografts were treated with ${ }^{131} \mathrm{I}$-PARPi via implanted osmotic pumps (Fig. 5). Uptake and retention of ${ }^{131}$ I-PARPi in these mice were high, as determined via periodic SPECT/CT measurement for up to $6 \mathrm{~d}$ after implantation. Healthy mice showed no such retention. Dosimetry was calculated on the basis of the SPECT/CT data, indicating that 9.1 Gy were delivered to the whole brain of a tumorbearing animal, whereas less than $1 \mathrm{~Gy}$ was delivered to a healthy animal. This shows that CED delivers its radiotherapeutic dose in such a way that PARP-overexpressing cells will retain the activity while the drug is cleared quickly from healthy brain cells (7).

\section{CONCLUSION}

We have identified PARP-1 as a potential anchor for radiotherapeutic PARP inhibitors in glioblastoma. We explored and validated the pharmacokinetics and pharmacodynamics of ${ }^{131}$ I-PARPi and showed that animals treated with the radiotherapeutic have a survival benefit, and healthy brain cells do not retain the radiotherapeutic. Future studies will need to show whether ${ }^{131}$ I-PARPi demonstrates an improvement in therapeutic efficacy over the existing standard of care.

\section{DISCLOSURE}

This work was supported by National Institutes of Health grants 1 R01 CA204441 (Thomas Reiner) and P30 CA008748. The authors thank the Tow Foundation and Memorial Sloan Kettering Cancer Center's Center for Molecular Imaging \& Nanotechnology (Brandon Carney and Susanne Kossatz), the National Science Foundation Integrative Graduate Education and Research Traineeship (IGERT 0965983 at Hunter College; Brandon Carney), the American-Italian Cancer Foundation (Giuseppe Carlucci), and the Imaging and Radiation Sciences Program (Thomas Reiner) for financial support. No other potential conflict of interest relevant to this article was reported.

\section{ACKNOWLEDGMENTS}

We thank the support of Memorial Sloan Kettering Cancer Center's Animal Imaging Core Facility, Radiochemistry \& Molecular Imaging Probes Core Facility, and Molecular Cytology Core Facility. In addition, we thank Dr. Ira Dunkel for helpful discussions and Leah Bassity for editing the manuscript.

\section{REFERENCES}

1. Alexander BM, Cloughesy TF. Adult glioblastoma. J Clin Oncol. 2017;35:24022409.

2. Bi WL, Beroukhim R. Beating the odds: extreme long-term survival with glioblastoma. Neuro-oncol. 2014;16:1159-1160.

3. Holland EC. Glioblastoma multiforme: the terminator. Proc Natl Acad Sci USA. 2000;97:6242-6244.

4. Park JK, Hodges T, Arko L, et al. Scale to predict survival after surgery for recurrent glioblastoma multiforme. J Clin Oncol. 2010;28:3838-3843.

5. Anjum K, Shagufta BI, Abbas SQ, et al. Current status and future therapeutic perspectives of glioblastoma multiforme (GBM) therapy: a review. Biomed Pharmacother. 2017;92:681-689.

6. Vogelbaum MA, Aghi MK. Convection-enhanced delivery for the treatment of glioblastoma. Neuro-oncol. 2015;17(suppl 2):ii3-ii8.

7. Luther N, Zhou Z, Zanzonico P, et al. The potential of theragnostic ${ }^{124} \mathrm{I}-8 \mathrm{H} 9$ convection-enhanced delivery in diffuse intrinsic pontine glioma. Neuro-oncol. 2014;16:800-806.

8. Parker NR, Khong P, Parkinson JF, Howell VM, Wheeler HR. Molecular heterogeneity in glioblastoma: potential clinical implications. Front Oncol. 2015;5:55.

9. Pommier Y, O'Connor MJ, de Bono J. Laying a trap to kill cancer cells: PARP inhibitors and their mechanisms of action. Sci Transl Med. 2016;8: 362 ps 17.

10. Kossatz S, Carney B, Schweitzer M, et al. Biomarker-based PET imaging of diffuse intrinsic pontine glioma in mouse models. Cancer Res. 2017;77:2112-2123.

11. Murnyák B, Kouhsari MC, Hershkovitch R, et al. PARP1 expression and its correlation with survival is tumour molecular subtype dependent in glioblastoma. Oncotarget. 2017;8:46348-46362.

12. Carney B, Kossatz S, Reiner T. Molecular imaging of PARP. J Nucl Med. 2017;58:1025-1030.

13. Carlucci G, Carney B, Brand C, et al. Dual-modality optical/PET imaging of PARP1 in glioblastoma. Mol Imaging Biol. 2015;17:848-855.

14. Salinas B, Irwin CP, Kossatz S, et al. Radioiodinated PARP1 tracers for glioblastoma imaging. EJNMMI Res. 2015;5:123-137.

15. Knight JC, Koustoulidou S, Cornelissen B. Imaging the DNA damage response with PET and SPECT. Eur J Nucl Med Mol Imaging. 2017;44:1065-1078. 
16. Irwin CP, Portorreal Y, Brand C, et al. PARPi-FL: a fluorescent PARP1 inhibitor for glioblastoma imaging. Neoplasia. 2014;16:432-440.

17. Reiner T, Lacy J, Keliher EJ, et al. Imaging therapeutic PARP inhibition in vivo through bioorthogonally developed companion imaging agents. Neoplasia. 2012; 14:169-177.

18. Carney B, Carlucci G, Salinas B, et al. Non-invasive PET imaging of PARP1 expression in glioblastoma models. Mol Imaging Biol. 2016;18:386-392.

19. Brown JA, Marala RB. Development of a high-throughput screening-amenable assay for human poly(ADP-ribose) polymerase inhibitors. J Pharmacol Toxicol Methods. 2002;47:137-141.

20. Dogdas B, Stout D, Chatziioannou AF, Leahy RM. Digimouse: a 3D whole body mouse atlas from CT and cryosection data. Phys Med Biol. 2007;52:577-587.

21. Sato T, Niita K, Matsuda N, et al. Particle and heavy ion transport code system, PHITS, version 2.52. J Nucl Sci Technol. 2013;45:634-638.

22. Zmuda F, Malviya G, Blair A, et al. Synthesis and evaluation of a radioiodinated tracer with specificity for poly(ADP-ribose) polymerase-1 (PARP-1) in vivo. J Med Chem. 2015;58:8683-8693.

23. Makvandi M, Xu K, Lieberman BP, et al. A radiotracer strategy to quantify PARP-1 expression in vivo provides a biomarker that can enable patient selection for PARP inhibitor therapy. Cancer Res. 2016;76:4516-4524.

24. Anderson RC, Makvandi M, Xu K, et al. Iodinated benzimidazole PARP radiotracer for evaluating PARP1/2 expression in vitro and in vivo. Nucl Med Biol. 2016;43:752-758.

25. Menear KA, Adcock C, Boulter R, et al. 4-[3-(4-cyclopropanecarbonylpiperazine1-carbonyl)-4-fluorobenzyl]-2H-phthalazin-1-one: a novel bioavailable inhibitor of poly(ADP-ribose) polymerase-1. J Med Chem. 2008;51:6581-6591.
26. Carney B, Lok BH, Schneeberger VE, et al. Target engagement imaging of PARP inhibitors in small cell lung cancer. Nat Commun. 2018;9:176.

27. Bianco AC, Kim BW. Deiodinases: implications of the local control of thyroid hormone action. J Clin Invest. 2006;116:2571-2579.

28. Brans B, Monsieurs M, Laureys G, Kaufman JM, Thierens H, Dierckx RA. Thyroidal uptake and radiation dose after repetitive I-131-MIBG treatments: influence of potassium iodide for thyroid blocking. Med Pediatr Oncol. 2002;38:41-46.

29. Doubrovin M, Ponomarev V, Beresten T, et al. Imaging transcriptional regulation of p53-dependent genes with positron emission tomography in vivo. Proc Natl Acad Sci USA. 2001;98:9300-9305.

30. Vardi Y, Ying Z, Zhang C-H. Two-sample tests for growth curves under dependent right censoring. Biometrika. 2001;88:949-960.

31. Bianco J, Bastiancich C, Jankovski A, des Rieux A, Préat V, Danhier F. On glioblastoma and the search for a cure: where do we stand. Cell Mol Life Sci. 2017;74:2451-2466.

32. Begg AC, Stewart FA, Vens C. Strategies to improve radiotherapy with targeted drugs. Nat Rev Cancer. 2011;11:239-253.

33. Thurber GM, Yang KS, Reiner T, et al. Single-cell and subcellular pharmacokinetic imaging allows insight into drug action in vivo. Nat Commun. 2013; 4:1504-1513.

34. Schinkel AH. P-glycoprotein, a gatekeeper in the blood-brain barrier. Adv Drug Deliv Rev. 1999;36:179-194.

35. Vaidyanathan A, Sawers L, Gannon AL, et al. ABCB1 (MDR1) induction defines a common resistance mechanism in paclitaxel- and olaparib-resistant ovarian cancer cells. Br J Cancer. 2016;115:431-441. 